\title{
Clinical Outcome and Underlying Genetic Cause of Functional Terminal Complement Pathway Deficiencies in a Multicenter UK Cohort
}

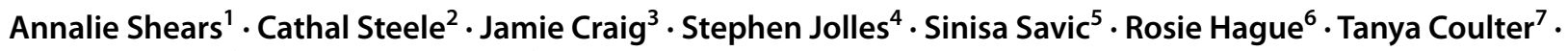 \\ Richard Herriot $^{8} \cdot$ Peter D. Arkwright $^{9}$
}

Received: 20 November 2021 / Accepted: 11 January 2022 / Published online: 27 January 2022

(c) The Author(s) 2022

\begin{abstract}
Background Terminal complement pathway deficiencies often present with severe and recurrent infections. There is a lack of good-quality data on these rare conditions. This study investigated the clinical outcome and genetic variation in a large UK multi-center cohort with primary and secondary terminal complement deficiencies.

Methods Clinicians from seven UK centers provided anonymised demographic, clinical, and laboratory data on patients with terminal complement deficiencies, which were collated and analysed.

Results Forty patients, median age 19 (range 3-62) years, were identified with terminal complement deficiencies. Ten (62\%) of 16 patients with low serum C5 concentrations had underlying pathogenic $C F H$ or $C F I$ gene variants. Two-thirds were from consanguineous Asian families, and $80 \%$ had an affected family member. The median age of the first infection was 9 years. Forty-three percent suffered meningococcal serotype B and $43 \%$ serotype Y infections. Nine (22\%) were treated in intensive care for meningococcal septicaemia. Two patients had died, one from intercurrent COVID-19. Twenty-one (52\%) were asymptomatic and diagnosed based on family history. All but one patient had received booster meningococcal vaccines and $70 \%$ were taking prophylactic antibiotics.

Discussion The genetic etiology and clinical course of patients with primary and secondary terminal complement deficiency are variable. Patients with low antigenic C5 concentrations require genetic testing, as the low level may reflect consumption secondary to regulatory defects in the pathway. Screening of siblings is important. Only half of the patients develop septicaemia, but all should have a clear management plan.
\end{abstract}

Keywords Terminal complement pathway $\cdot$ Factor $\mathrm{H} \cdot$ Factor I $\cdot$ Meningococcal infection $\cdot$ Genetics

Peter D. Arkwright

peter.arkwright@nhs.net

1 NIHR Paediatric Academic Clinical Fellow, University of Manchester, Manchester, UK

2 NHS Tayside, Ninewells Hospital and Medical School, Dundee, Scotland

3 University of Dundee, Dundee, Scotland

4 University Hospital of Wales, Cardiff, Wales

5 School of Medicine, University of Leeds, Leeds, \& NIHRBRC, Leeds, UK

6 Immunology, Royal Hospital for Children, Glasgow, Scotland

7 Immunology, Belfast Health and Social Care Trust, Belfast, Northern Ireland

8 Immunology, Aberdeen Royal Infirmary, Aberdeen, Scotland

9 Paediatric Immunology, Lydia Becker Institute, University of Manchester, Manchester, UK

\section{Abbreviations \\ $\mathrm{CFH}$ Complement factor $\mathrm{H}$ \\ CHI Complement factor I \\ MAC Membrane attack complex}

\section{Introduction}

The complement system is an important component of innate immune defence against invading bacteria, particularly encapsulated meningococci and pneumococci. Terminal complement deficiencies are inherited as autosomal recessive traits and their prevalence varies, depending on consanguinity and family size [1]. The terminal complement pathway is made up of five components (C5-C9). They combine to form the pore-forming membrane attack complex (MAC) that disrupts membranes and lyse bacteria [2]. 
Deficiencies in terminal complement components impair immunity, particularly against meningococci [3]. Patients have a 7,000 to 10,000-fold higher risk of invasive meningococcal disease, and half have recurrent infections $[4,5]$. Less prevalent meningococcal strains with lower virulence often cause invasive meningococcal infections in these patients $[4,6]$.

Good-quality data on this topic are sparse, with only a few published case reports or series [6-11]. The aim of this study was to characterise the clinical and laboratory features of a large UK cohort of patients with primary and secondary terminal complement deficiencies, to understand more fully the morbidity and mortality, thus providing information to guide future management decisions.

\section{Methods}

\section{Patient Identification and Recruitment}

This was a retrospective cohort study, in which clinicians working in major UK clinical immunology referral centers were emailed and invited to participate. Seven centers participated, with an overall response rate of $80 \%$. Patients with primary or secondary terminal pathway complement immunodeficiencies defined by undetectable AP50 levels were identified from local databases at the referring clinical immunology center. Only patients where C5, C6, C7, $\mathrm{C} 8$, and $\mathrm{C} 9$ complement concentrations had been measured and a low level found were included. Ethics approval was obtained from the National UK Ethics Committee (IRAS number 259418).

\section{Data Collection and Analysis}

Anonymised data containing no patient identifiers were entered into standardised Excel data sheets by the clinician with details of demographic, clinical, and laboratory parameters (CH50, AP50 and specific complement factors, genetic mutation screening, vaccine responses) and treatment received (vaccines, antibiotics, intensive care admissions).

Data were collated and analysed using IBM SPSS Statistics 25. Continuous variables are quoted as medians and ranges. For discrete variables, groups were compared using the chi-square test. Multivariate analysis was performed using binominal logistic regression. Differences were considered statistically significant if the $p$ value was $<0.05$.

\section{Complement Assays}

Complement measurements were performed by accredited regional immunology laboratories using standard techniques [12]. Briefly, complement-dependent lysis of antibody-sensitised red blood cells in either a fluid or gel was used to provide a quantitative measure of functional activity ( $\mathrm{CH} 50$ for the classical pathway and AP50 for the alternative pathway). Specific complement components of the alternate pathway and $\mathrm{C} 5$ to $\mathrm{C} 9$, or factor $\mathrm{H}$ and I were detected by either gel diffusion (Ouchterlony technique) or ELISA. Subsequent gene sequencing was initiated by physicians liaising with the UK accredited regional clinical genetics centers.

\section{Results}

\section{Demographics}

Forty patients with primary and secondary terminal complement deficiencies were identified from seven clinical immunology centers across the UK (Aberdeen, Belfast, Cardiff, Dundee, Glasgow, Leeds, Manchester) (Table 1, Table SI). Twenty-two (55\%) patients were male. The median age at the last appointment or death was 19 (3-62) years. Ethnic backgrounds were white European $(13,32 \%)$ or Asian $(27,68 \%)$. Eighty percent

Table 1 Demographic characteristics of patients with terminal complement pathway deficiencies

\begin{tabular}{|c|c|c|c|c|c|c|c|}
\hline & Total cohort & CF5 & CF6 & CF7 & CF8 & $\mathrm{CFH}$ & $\mathrm{CFI}$ \\
\hline Number & 40 & $6(15 \%)$ & $6(15 \%)$ & $9(22.5 \%)$ & $9(22.5 \%)$ & $2(5 \%)$ & $8(20 \%)$ \\
\hline Age at last review/death (years) & $19(3-62)$ & $17(3-20)$ & $23(16-62)$ & $19(9-40)$ & $30(6-60)$ & $6(3-8)$ & $18(7-30)$ \\
\hline Age at diagnosis (years) & $14(1-45)$ & $8(1-16)$ & $16(12-30)$ & $15(1-45)$ & $24(8-27)$ & $10(7-14)$ & $10(3-16)$ \\
\hline Gender (male) & $22(55 \%)$ & $4(67 \%)$ & $3(50 \%)$ & $5(56 \%)$ & $5(56 \%)$ & $1(50 \%)$ & $4(50 \%)$ \\
\hline \multicolumn{8}{|l|}{ Ethnicity } \\
\hline White European & $13(32 \%)$ & $2(33 \%)$ & $2(33 \%)$ & $2(22 \%)$ & $5(56 \%)$ & $2(100 \%)$ & - \\
\hline Asian & $27(68 \%)$ & $4(67 \%)$ & $4(67 \%)$ & $7(78 \%)$ & $4(44 \%)$ & - & $8(100 \%)$ \\
\hline Consanguinity & $26(65 \%)$ & $4(67 \%)$ & $3(50 \%)$ & $7(78 \%)$ & $4(44 \%)$ & - & $8(100 \%)$ \\
\hline Family History & $32(80 \%)$ & $5(83 \%)$ & $3(50 \%)$ & $9(100 \%)$ & $5(56 \%)$ & $2(100 \%)$ & $8(100 \%)$ \\
\hline
\end{tabular}

Discrete variables, numbers (\%); age, median (range) 
had a family history of a terminal complement deficiency, and $26(65 \%)$ had known consanguineous parenthood. All patients had undetectable AP50.

Six $(15 \%)$ patients were deficient in C5, six $(15 \%)$ in C6, nine $(22.5 \%)$ in $\mathrm{C} 7$, and nine $(22.5 \%)$ in $\mathrm{C} 8$. Ten patients $(25 \%)$ who had a low C5 concentration were subsequently found on genetic testing to have primary complement factor $\mathrm{H}$ or I, rather than C5 deficiency (Fig. 1).

\section{Clinical Infections}

Nineteen (48\%) patients had a history of meningococcal and/ or other deep-seated bacterial infections. Eighteen (45\%) patients suffered from meningococcal septicaemia, seven (18\%) meningococcal meningitis, three (8\%) meningococcal arthritis, and one (2\%) meningococcal Fitz-Hugh Curtis syndrome. The median age at first meningococcal infection was 9 years (1-25 years), and the median number of infections was one (range 0-5) (Table 2). Of patients who developed

Fig. 1 Distribution of primary and secondary terminal complement component deficiencies

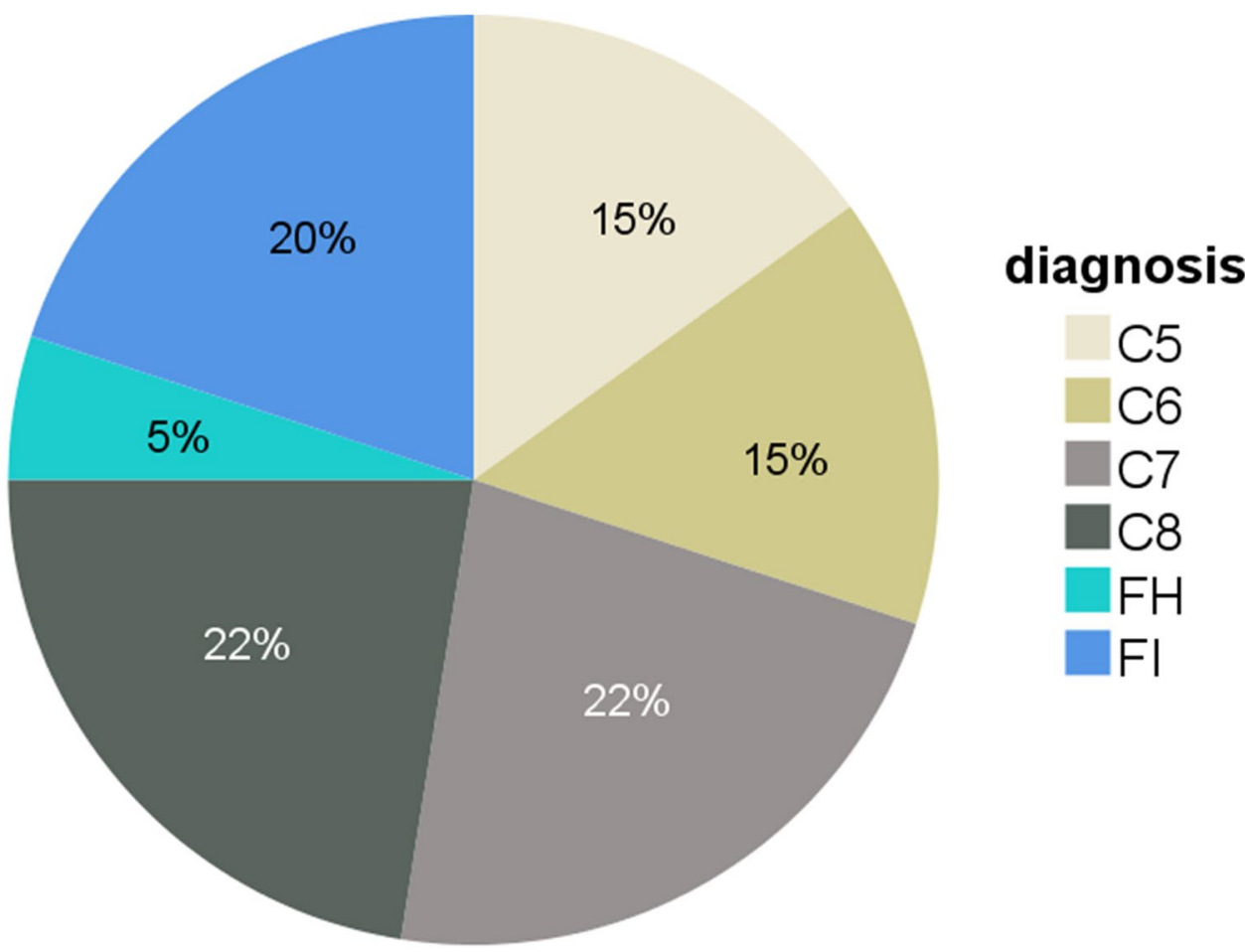

Table 2 Meningococcal infections and mortality

\begin{tabular}{|c|c|c|c|c|c|c|c|}
\hline & $\begin{array}{l}\text { Total cohort } \\
40\end{array}$ & $\begin{array}{l}\text { CF5 } \\
6(15 \%)\end{array}$ & $\begin{array}{l}\text { CF6 } \\
6(15 \%)\end{array}$ & $\begin{array}{l}\text { CF7 } \\
9(22.5 \%)\end{array}$ & $\begin{array}{l}\text { CF8 } \\
9(22.5 \%)\end{array}$ & $\begin{array}{l}\mathrm{CFH} \\
2(5 \%)\end{array}$ & $\begin{array}{l}\text { CFI } \\
8(20 \%)\end{array}$ \\
\hline No. with no infection history & $21(52 \%)$ & $4(67 \%)$ & $2(33 \%)$ & $7(78 \%)$ & $3(33 \%)$ & 0 & $5(62 \%)$ \\
\hline Age at first infection (years) & $9(1-25)$ & $4(0-10)$ & $14(4-15)$ & $15(8-16)$ & $7(1-25)$ & $8(1-14)$ & $7(1-10)$ \\
\hline No. of meningococcal infections & $1(0-5)$ & $0(0-5)$ & $2(0-3)$ & $0(0-2)$ & $2(0-4)$ & $2(1-2)$ & $0(0-2)$ \\
\hline Meningococcal serotypes & 14 & 4 & 4 & 3 & 1 & 1 & 1 \\
\hline $\mathrm{B}$ & $6(43 \%)$ & $2(50 \%)$ & $2(40 \%)$ & $1(50 \%)$ & - & - & $1(100 \%)$ \\
\hline $\mathrm{E}$ & $1(7 \%)$ & - & - & - & $1(100 \%)$ & - & - \\
\hline W & $1(7 \%)$ & - & $1(40 \%)$ & - & - & - & - \\
\hline $\mathrm{Y}$ & $6(43 \%)$ & $2(50 \%)$ & $1(20 \%)$ & $2(50 \%)$ & - & $1(100 \%)$ & - \\
\hline Septicemia & $18(45 \%)$ & 2 & 4 & 2 & 5 & 2 & 3 \\
\hline Meningitis & $7(18 \%)$ & - & 3 & 1 & 3 & - & - \\
\hline Arthritis & $3(8 \%)$ & - & 1 & - & 1 & - & 1 \\
\hline Fitz-Hugh Curtis syndrome & $1(2 \%)$ & - & - & - & 1 & - & - \\
\hline Non-meningococcal sepsis & $14(34 \%)$ & $2(33 \%)$ & $3(50 \%)$ & $1(10 \%)$ & $3(33)$ & $1(50 \%)$ & $4(50 \%)$ \\
\hline Deaths & $2(5 \%)$ & - & - & - & 1 & - & 1 \\
\hline
\end{tabular}

Discrete variables: numbers (\%); age, median (range) 
meningococcal infections for which serotypes were identified (14 (67\%) of 21), the majority were B $(6,43 \%)$ or Y $(6$, $43 \%)$. Serotypes $\mathrm{W}(1,7 \%)$ and $\mathrm{E}(1,7 \%)$ were also identified (Fig. 2). Non-meningococcal bacterial infections were observed in a third of patients, including upper and lower respiratory infections (7.5\%), pyogenic meningitis $(7.5 \%)$, osteomyelitis/septic arthritis (7.5\%), and skin/soft tissue infections (10.0\%) (Table SII). Two males (5\%) died, one at 4 months old of pneumococcal meningitis, and another at 60 years old of COVID-19 not thought to be related to his complement deficiency.

All patients without a family history of complement deficiency had suffered from meningococcal sepsis, while only $35 \%$ of patients with a family history developed sepsis $(p<0.001)$. Presentation with meningococcal sepsis was a major risk factor for admission to intensive care (relative risk $16.3,95 \%$ confidence interval: $1.5-186)$. The risk of meningococcal septicaemia and admission to intensive care was not correlated with genetic diagnosis, age, gender, or antibiotic prophylaxis.

\section{Laboratory Findings}

The results of genetic testing were available on $34(85 \%)$ of the 40 patients (Table SI). Twenty-five (62.5\%) had pathogenic homozygous gene variants, while in nine $(22.5 \%)$ patients gene variants were compound heterozygous. Sixtynine percent of patients with a history of consanguinity had a homozygous gene variant, compared with $36 \%$ of patients with no history $(p<0.05)$. Ten patients had antigenically low C5, but on genetic testing, eight had CFI gene mutations and two a $\mathrm{CFH}$ gene mutation indicating excessive $\mathrm{C} 5$ consumption rather than defective production.

\section{Management}

Nine patients (22\%) were admitted to the intensive care unit, and two patients were given FFP for fulminant meningococcaemia (Table 3). Ninety-eight percent of patients were given additional MenB/ MenACWY vaccines after diagnosis. Six patients (15\%) also received an extra conjugate pneumococcal vaccine. Seventy percent of patients were prescribed either amoxicillin or penicillin prophylaxis, although $22 \%$ admitted to being noncompliant with antibiotic prophylaxis. The rate of compliance did not correlate with age, gender, ethnicity, intensive care admission, meningococcal septicemia, or meningitis.

\section{Discussion}

This is the largest retrospective, multicenter, cohort study of patients with terminal complement deficiencies. There are several lessons to be learned from the study.

Two-thirds of patients were from a consanguineous Asian family and $80 \%$ had an affected family member, highlighting the importance of testing siblings of index cases. The only patient to die as a direct complication of his complement deficiency (factor I) was a 4-month-old who succumbed to fulminant pneumococcal meningitis. The infant had three older siblings who were regularly being followed by the clinical immunology service. However, the immunology team was not made aware of the
Fig. 2 Meningococcal infection by serotype. The relative percentages of each serotypes associated with invasive meningococcal disease in England between 1999 and 2019 are quoted in brackets in the legend [13]

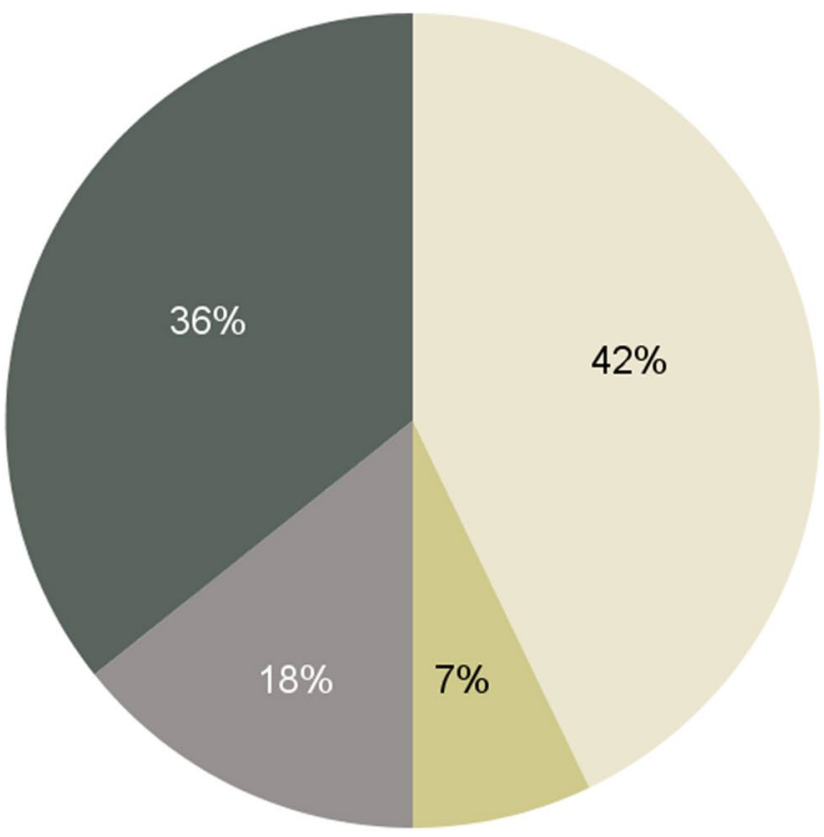

meningococcal serotype

B (50-60\%)

$E(<1 \%)$

$\mathrm{W}(3-21 \%)$

$Y(1-11 \%)$ 
Table 3 Clinical management

\begin{tabular}{llllllll}
\hline & Total cohort & CF5 & CF6 & CF7 & CF8 & CFH & CFI \\
& 40 & $6(15 \%)$ & $6(15 \%)$ & $9(22.5 \%)$ & $9(22.5 \%)$ & $2(5 \%)$ & $8(20 \%)$ \\
\hline Booster vaccinations & $39(98 \%)$ & 6 & 6 & 9 & 9 & 2 & 7 \\
MenB/ACWY & $6(15 \%)$ & 1 & - & - & 1 & - & 4 \\
PCV7/13 & $3(8 \%)$ & 1 & - & - & 1 & - & 1 \\
Hib & & & & & & & \\
Vaccine responses checked & $36(90 \%)$ & 6 & 3 & 9 & 9 & 2 & 7 \\
Antibiotic prophylaxis & $11(28 \%)$ & 1 & 4 & - & 5 & - & 1 \\
Penicillin V & $17(42 \%)$ & 4 & - & 7 & 2 & 1 & 3 \\
Amoxicillin & $2(5 \%)$ & - & - & - & 1 & - & 1 \\
None & $1(2 \%)$ & 1 & - & - & - & - & - \\
Unknown & $10(22 \%)$ & 1 & 2 & 2 & 1 & 1 & 3 \\
Non-compliant & & & & & & & 2 \\
Intensive care admission & $9(22 \%)$ & 3 & 2 & - & 2 & - & 2 \\
Fresh frozen plasma & $2(5 \%)$ & 1 & - & - & - & - & 1 \\
\hline
\end{tabular}

For discrete variables: numbers (\%)

mother's pregnancy nor the child's birth. This tragic case illustrates the importance of ensuring early screening and a clear management plan for infants born to families with immune deficiency. A previous UK study screened 824 patients who had previously suffered from meningococcal sepsis for primary complement deficiency and found only one case [14]. Unlike their series, many of our cohorts had consanguineous parents and other affected siblings, highlighting the importance of taking a thorough family history in patients with meningococcal disease (especially with less common serotypes) or unusual or recurrent infections.

DNA was sent for genetic sequencing in the majority of patients, particularly those with low serum C5 concentrations. Ten (62\%) of 16 patients with low C5 antigenemia had $C F H$ or $C F I$ pathogenic gene variants with normal C5 genetics, consistent with a diagnosis of secondary consumptive $\mathrm{C} 5$ deficiency due to a primary defect in the regulatory complement component. We recommend that all patients with a suspected complement deficiency should have confirmatory genetic testing. Complement factors $\mathrm{H}$ and I regulate the formation of $\mathrm{C} 3$ and $\mathrm{C} 5$ convertase enzymes and cause excessive consumption [15]. In contrast, to complete deficiencies of complement factors suffered by patients in our cohort, haplo-insufficiencies of these regulatory factors are often associated with normal serum protein levels and overactivity of the complement pathway, leading to atypical haemolytic uremic syndrome or other vasculopathies [16, 17]. In addition, those patients treated with anti-C5 biologics such as eculizumab or ravulizumab also have a higher risk of meningococcaemia [18]. No C9 deficient patients were detected in this series, in keeping with the absence of this immune deficiency in the UK and other European Primary Immunodeficiency registries [19]. In contrast, C9 deficiency has been documented in some Japanese patients with terminal complement deficiency [20].
This is one of the first studies to document the proportion of family members with terminal complement deficiency who are asymptomatic. Fifty-two percent of patients (median age of 17 (range 3-46) years) had no prior history of bacterial sepsis. Asymptomatic adults cannot be excluded based on the absence of clinical disease and their complement pathway should be tested. It is also important to be aware that the median age of first meningococcal infection in these patients was 9 (range 1-25) years, while in the UK, the incidence of meningococcal infections is highest in infants under one year old [21]. In keeping with our findings, Platonov et al. previously found that the mean age of first meningococcal infection in their cohort of terminal complement deficient patients was 15 years old [22]. Thus, there should be a higher index of suspicion of complement defects in patients presenting later in childhood or beyond.

Patients with defects in terminal complement deficiencies typically suffer from meningococcal sepsis as was the case with nearly half of the patients in this cohort [23]. The relatively low mortality per episode of meningococcal disease in patients with terminal complement deficiencies has been demonstrated previously, hypothesised as due to lower endotoxin release from the bacterial surface in the absence of a complete terminal complement pathway [22]. Deep-seated non-meningococcal infections are also common, occurring in one-third of patients, and include bacterial meningitis, osteomyelitis/septic arthritis, sinopulmonary infection, and soft-tissue infections.

Conjugate meningococcal B and ACYW and pneumococcal and Haemophilus influenzae type B vaccines should be given to all patients with complement deficiencies, with subsequent monitoring of antibody titres. Prophylactic antibiotics are recommended, particularly in patients with recurrent meningococcal infections or those at higher risk of endemic or occupational exposure [24]. The use of prophylactic antibiotics should be balanced against the potential development 
of resistance. Patients also need education, including an emergency plan in the event of infection and travel. Some patients have emergency antibiotics at home and MedicAlert or similar bracelets. The effectiveness of these measures in preventing disease is not possible to determine from the present study. This study found no association between either rate of infection or admission to intensive and receiving antibiotic prophylaxis but did not formally assess adherence. Twenty-two percent of patients admitted not to take their antibiotic prophylaxis, but there were no obvious predictive demographic or clinical correlates. Adherence and factors influencing it are generic issues that would be possibly better addressed in studies of adherence to medications given for more common conditions, e.g., asthma, hypertension, and HIV.

In summary, this study highlights the complexities of terminal complement deficiencies. Meningococcal infection over 5 years of age and with less common serotypes (e.g. $\mathrm{E}, \mathrm{Y}$ ) or recurrent meningococcal infection should prompt further investigation of terminal pathway defects, particularly where there is a family history of unusual or deepseated bacterial infections or consanguinity. The importance of genetic testing for possible factor $\mathrm{H}$ and I deficiency in patients with low antigenic C5 levels is also emphasised. Further studies investigating the prevalence of terminal complement deficiencies and the impact of antibiotic prophylaxis and vaccination on outcome are required. The use of international databases may enable long-term follow-up of these patients.

Supplementary Information The online version contains supplementary material available at https://doi.org/10.1007/s10875-022-01213-9.

Author Contribution CS and PDA conceived the study. AS and PDA collated and analysed the data supplied by the other co-authors and drafted the manuscript. All authors critically appraised and gave feedback on the final version.

Funding AS is a NIHR Academic Clinical Fellow. The National Institute for Health Research (NIHR) using UK aid from the UK Government supports global health research.

Data Availability Data is available on request to PDA, the corresponding author.

Code Availability Not applicable.

\section{Declarations}

Ethics Approval Ethics approval was obtained from the National UK Ethics Committee (IRAS number 259418).

Consent to Participate Written consent was obtained from patients or their parents.

Consent for Publication Ethics approval and written consent included consent for publication.
Conflict of Interest The authors declare no competing interests.

Open Access This article is licensed under a Creative Commons Attribution 4.0 International License, which permits use, sharing, adaptation, distribution and reproduction in any medium or format, as long as you give appropriate credit to the original author(s) and the source, provide a link to the Creative Commons licence, and indicate if changes were made. The images or other third party material in this article are included in the article's Creative Commons licence, unless indicated otherwise in a credit line to the material. If material is not included in the article's Creative Commons licence and your intended use is not permitted by statutory regulation or exceeds the permitted use, you will need to obtain permission directly from the copyright holder. To view a copy of this licence, visit http://creativecommons.org/licenses/by/4.0/.

\section{References}

1. El Sissy C, Rosain J, Vieira-Martins P, Bordereau P, Gruber A, Devriese $\mathrm{M}$, et al. Clinical and genetic spectrum of a large cohort with total and Sub-total complement deficiencies. Front Immunol. 2019;10:1936.

2. Krawczyk PA, Laub M, Kozik P. To kill but not be killed: controlling the activity of mammalian pore-forming proteins. Front Immunol. 2020;11:601405.

3. Arkwright PD 2021 Chapter 18. Primary immunodeficiencies of complement. In: Primary and secondary immunodeficiency: a case-based guide to evaluation and management. Ed Jonathan Bernstein, Springer Nature Switzerland AG, 313-330.

4. Turley AJ, Gathmann B, Bangs C, Bradbury M, Seneviratne S, Gonzalez-Granado LI, Hackett S, Kutukculer N, et al. Spectrum and management of complement immunodeficiencies (excluding hereditary angioedema) across Europe. J Clin Immunol. 2015;35:199-205.

5. Ladhani SN, Campbell H, Lucidarme J, Gray S, Parikh S, Willerton L, Clark SA, Lekshmi A, et al. Invasive meningococcal disease in patients with complement deficiencies: a case series (2008-2017). BMC Infect Dis. 2019;14(19):522.

6. Rosain J, Hong E, Fieschi C, Martins PV, El Sissy C, Deghmane AE, et al. Strains responsible for invasive meningococcal disease in patients with terminal complement pathway deficiencies. J Infect Dis. 2017;215:1331-8.

7. Rauscher CK, Fajt ML, Bryk J, Petrov AA. Clinical implications of C6 complement component deficiency. Allergy Asthma Proc. 2020;41:386-8.

8. Owen EP, Würzner R, Leisegang F, Rizkallah P, Whitelaw A, Simpson J, Thomas AD, Harris CL, et al. A complement C5 gene mutation, c.754G>A:p.A252T, is common in the Western Cape, South Africa and found to be homozygous in seven percent of Black African meningococcal disease cases. Mol Immunol. 2015;64:170-6.

9. Owen EP, Würzner R, Leisegang F, Rizkallah P, Whitelaw A, Simpson J, et al. A complement C5 gene mutation, c.754G $>$ A:p. A252T, is common in the Western Cape, South Africa and found to be homozygous in seven percent of Black African meningococcal disease cases. Mol Immunol. 2015;64:170-6.

10. Rameix-Welti MA, Régnier CH, Bienaimé F, Blouin J, Schifferli J, Fridman WH, Sautès-Fridman C, et al. Hereditary complement C7 deficiency in nine families: subtotal C7 deficiency revisited. Eur J Immunol. 2007;37:1377-85.

11. Audemard-Verger A, Descloux E, Ponard D, Deroux A, Fantin $\mathrm{B}$, Fieschi C, et al. Infections revealing complement deficiency in adults: a French nationwide study enrolling 41 patients. Medicine. 2016;95:e3548. 
12. Willrich MA Braun KM, Moyer AM, Jeffrey DH, Frazer-Abel A 2021 Complement testing in the clinical laboratory. Crit Rev Lab Sci 1-51.

13. Public Health England. Invasive meningococcal disease in England: annual laboratory confirmed reports for epidemiological year 2018 to 2019. Health Prot Rep. 2019;13:38.

14. Hoare S, El-Shazali O, Clark JE, Fay A, Cant AJ. Investigation for complement deficiency following meningococcal disease. Arch Dis Child. 2002;86:215-7.

15. Ferreira VP, Pangburn MK, Cortés C. Complement control protein factor $\mathrm{H}$ : the good, the bad, and the inadequate. Mol Immunol. 2010;47:2187-97.

16. Alba-Domínguez M, López-Lera A, Garrido S, Nozal P, González-Granado I, Melero J, et al. Complement factor I deficiency: a not so rare immune defect Characterization of new mutations and the first large gene deletion. Orphanet J Rare Dis. 2012;7:42.

17. Wong EK, Kavanagh D. Diseases of complement dysregulationan overview. Semin Immunopathol. 2018;40:49-64.

18. Mbaeyi SA, Bozio CH, Duffy J, Rubin LG, Hariri S, Stephens DS, et al. Meningococcal vaccination: recommendations of the advisory committee on immunization practices, United States, 2020. MMWR Recomm Rep. 2020;69:1-41.

19. El-Helou SM, Biegner AK, Ehl SR, Heeg M, Maccari ME, Ritterbusch $\mathrm{H}$, et al. The German national registry of primary immunodeficiencies (2012-2017). Front Immunol. 2019;10:3389.
20. Nagata M, Hara T, Aokl T, Mizuno Y, Akeda H, Inaba S, et al. Inherited deficiency of ninth component of complement: an increased risk of meningococcal meningitis. J Pediatr. 1989;114:260-4.

21. Public Health England. Invasive meningococcal disease in England: annual laboratory confirmed reports for epidemiological year 2019 to 2020. Health Prot Rep. 2021;15:1-8.

22. Platonov AE, Beloborodov VB, Vershinina IV. Meningococcal disease in patients with later complement component deficiency: studies in the U.S.S.R. Medicine. 1993;93:374-92.

23. Ram S, Lewis LA, Rice PA. Infections of people with complement deficiencies and patients who have undergone splenectomy. Clin Microbiol Rev. 2010;23:740-80.

24. Brodszki N, Frazer-Abel A, Grumach AS, Kirschfink M, Litzman J, Perez E, et al. European society for immunodeficiencies (ESID) and European reference network on rare primary immunodeficiency, autoinflammatory and autoimmune diseases (ERN RITA) complement guideline: deficiencies, diagnosis, and management. J Clin Immunol. 2020;40:576-91.

Publisher's Note Springer Nature remains neutral with regard to jurisdictional claims in published maps and institutional affiliations. 